\title{
The flavonoid morin from Moraceae induces apoptosis by modulation of Bcl-2 family members and Fas receptor in HCT 116 cells
}

\author{
HWANG-BO HYUN ${ }^{1}$, WON SUP LEE ${ }^{2}$, SE-IL GO ${ }^{2}$, ARULKUMAR NAGAPPAN ${ }^{2}$, \\ CHEOL PARK $^{3}$, MIN HO HAN ${ }^{5}$, SU HYUN HONG ${ }^{5}$, GONSUP KIM ${ }^{6}$, GI YOUNG KIM ${ }^{9}$, \\ JAEHUN CHEONG ${ }^{10}$, CHUNG HO RYU ${ }^{7}$, SUNG CHUL SHIN ${ }^{8}$ and YUNG HYUN $\mathrm{CHOI}^{1,4}$ \\ ${ }^{1}$ Department of Biochemistry, Dongeui University College of Korean Medicine, Busan 614-052; \\ ${ }^{2}$ Department of Internal Medicine, Institute of Health Sciences, Gyeongsang National University School \\ of Medicine, Jinju 660-702; ${ }^{3}$ Department of Molecular Biology, College of Natural Sciences, and \\ ${ }^{4}$ Anti-Aging Research Center and Blue-Bio Industry RIC, Dongeui University, Busan 614-714; \\ ${ }^{5}$ Department of Biochemistry, Dongeui University College of Oriental Medicine, Busan 614-052; \\ ${ }^{6}$ School of Veterinary Medicine, ${ }^{7}$ Division of Applied Life Science (BK 21 Program) and \\ ${ }^{8}$ Department of Chemistry, Research Institute of Life Science, Gyeongsang National University, \\ Jinju 660-701; ${ }^{9}$ Laboratory of Immunobiology, Department of Marine Life Sciences, \\ Jeju National University, Jeju 690-756; ${ }^{10}$ Department of Molecular Biology, College of \\ Natural Sciences, Pusan National University, Busan 609-735, Republic of Korea
}

Received February 13, 2015; Accepted March 27, 2015

DOI: 10.3892/ijo.2015.2967

\begin{abstract}
It is evident based on literature that flavonoids from fruit can safely modulate cancer cell biology and induce apoptosis. Therefore, we investigated the anticancer activity of morin, a flavonoid which is plentiful in twigs of mulberry focusing on apoptosis, and its mechanisms. Morin upregulated the Fas receptor, and activates caspase- $8,-9$ and -3 in HCT-116 cells. Morin also activates Bid, and induced the loss of mitochondrial membrane potential (MMP, $\Delta \Psi_{\mathrm{m}}$ ) with Bax protein activation and cytochrome $\mathrm{c}$ release. In addition, morin induced ROS generation which was not blocked by $\mathrm{N}$-acetylcysteine. Morin also suppressed Bcl-2 and cIAP-1, anti-apoptotic proteins, which may contribute to augmentation of morin-triggered apoptosis. As an upstream signaling pathway, suppressed Akt activity by morin was asso-
\end{abstract}

Correspondence to: Professor Won Sup Lee, Department of Internal Medicine, Institute of Health Sciences and Gyeongnam Regional Cancer Center, Gyeongsang National University School of Medicine, 90 Chilam-dong, Jinju 660-702, Republic of Korea

E-mail: lwshmo@hanmail.net; lwshmo@gnu.ac.kr

Dr Yung Hyun Choi, Department of Biochemistry, Dongeui University College of Oriental Medicine, 42 San, Yangjung-dong, Busan 614-052, Republic of Korea

E-mail: choiyh@deu.ac.kr

Key words: morin, Bcl-2, Fas receptor, HCT-116 human colon cancer cells ciated to apoptosis. This study suggests that morin induces caspase-dependent apoptosis through extrinsic pathway by upregulating Fas receptor as well as through the intrinsic pathway by modulating Bcl-2 and IAP family members, and ROS generation, and that Akt is the critical upstream signaling that regulates the apoptotic effect of morin in human colon cancer HCT-116 cells.

\section{Introduction}

With advances in medical science, our lifespan has been extended. The population of elderly cancer patients and their cancer-related mortality are increasing $(1,2)$. These elderly patients with cancer have a lack of vital capacity, and cannot tolerate cytotoxic chemotherapy. Actually substantial portion of the elderly patients with cancer has experienced serious side effects from cytotoxic chemotherapy, and related complications. Therefore, changes in the chemotherapy approach are essential for better cancer treatment emphasizing quality of life. It is reported that high intake of fruits and vegetables may prevent cancer development and therefore attention has been drawn to the possibility of preventing or controlling cancer using flavonoids from fruits $(3,4)$. Furthermore, flavonoids in fruit can also enhance anticancer effects (5). Morin $\left(3,5,7,2^{\prime}, 4^{\prime}\right.$-pentahydroxyflavone) is a flavonoid originally isolated from members of the Moraceae family. It has been reported to possess certain properties that regulate the inflammatory response which leads to carcinogenesis arrest and cancer progression $(6,7)$.

Apoptosis is a process of programmed cell death with characteristic morphological changes, such as blebbing, cell 
shrinkage, nuclear fragmentation, chromatin condensation, and chromosomal DNA fragmentation (8). The flavonoids from fruits and vegetables show anticancer effects by inducing apoptosis. In addition, the apoptosis process eliminates the damaged cells which are susceptible to develop cancer and thereby serves as a defense mechanism for cancer development (9). These processes are regulated by a various range of cell signaling pathways. However, the mechanisms regarding morin-induced apoptosis in cancer cells are not fully elucidated especially regarding death receptor-mediated apoptosis. Here, we investigated the anticancer activity along with the mechanisms focusing on apoptosis in HCT-116 human colon cancer cells.

\section{Materials and methods}

Cells and reagents. HCT-116 human colon cancer cells from the American type culture collection (Rockville, MD, USA) were cultured in RPMI-1640 medium (Invitrogen Corp., Carlsbad, CA, USA) supplemented with $10 \%$ (v/v) fetal bovine serum (FBS) (Gibco BRL, Grand Island, NY, USA), $1 \mathrm{mM}$ L-glutamine, $100 \mathrm{U} / \mathrm{ml}$ penicillin, and $100 \mu \mathrm{g} / \mathrm{ml}$ streptomycin at $37^{\circ} \mathrm{C}$ in a humidified atmosphere of $95 \%$ air and $5 \% \mathrm{CO}_{2}$. Morin was obtained from Aging Tissue Bank (Pusan, Korea). Antibodies against Bcl-2 (N-19), Bax, Bid, t-Bid, cytochrmome c, BAD, TNF-related apoptosis-inducing ligand (TRAIL), TRAIL receptors (DR4, DR5), Fas receptor, FasL, X-linked inhibitor of apoptosis protein (XIAP), cellular inhibitor of apoptosis protein-1 (cIAP-1), cIAP-2, survivin, procaspase 3, procaspase 8 , and procaspase 9 were purchased from Santa Cruz Biotechnology (Santa Cruz, CA, USA). Antibody against poly(ADP-ribose) polymerase (PARP) was purchased from PharMingen (San Diego, CA, USA). Antibodies against phospho-ERK phospho-JNK, phosphop38 MAPK, p-Akt were purchased from Cell Signaling Technology, Inc. (Beverly, MA, USA). Peroxidase-labeled donkey anti-rabbit and sheep anti-mouse immunoglobulin, and an enhanced chemiluminescence (ECL) kit were purchased from Amersham (Arlington Heights, IL, USA). All other chemicals not specifically cited here were purchased from Sigma Chemical Co. (St. Louis, MO, USA). All the solutions were stored at $-20^{\circ} \mathrm{C}$. Propidium iodide (PI, $1 \mathrm{mg} / \mathrm{ml}$ ) was prepared in phosphate-buffered saline (PBS).

Cell viability assay. The cytotoxicity was determined by performing 3-(4, 5-dimethylthiazol-2-yl)-2, 5-diphenyltetrazolium bromide (MTT) assay and a tryphan blue exclusion method. For the MTT assay, cells were seeded at $10 \times 10^{4}$ cells/ $\mathrm{ml}$ in a 12-well plate and treated with morin for $48 \mathrm{~h}$. Following the treatments, $0.5 \mathrm{mg} / \mathrm{ml} \mathrm{3-(4,5-dimethylthiazol-}$ 2-yl)-2, 5-diphenyltetrazolium bromide $(0.5 \mathrm{mg} / \mathrm{ml})$ solution was added, prior to incubation for $3 \mathrm{~h}$ at $37^{\circ} \mathrm{C}$ in the dark. The absorbance of each well was measured at $540 \mathrm{~nm}$ with an enzyme-linked immunosorbent assay (ELISA) reader (Molecular Devices, LLC, Sunnyvale, CA, USA).

DNA fragmentation assay. After treatment with the indicated concentration of morin the cells were harvested and lysed in a buffer containing $10 \mathrm{mM}$ Tris- $\mathrm{HCl}(\mathrm{pH} 7.4), 150 \mathrm{mM}$ $\mathrm{NaCl}, 5 \mathrm{mM}$ EDTA, and $0.5 \%$ Triton $\mathrm{X}-100$ for $1 \mathrm{~h}$ at room temperature. The lysates were vortexed and cleared by centrifugation at $14,000 \mathrm{rpm}$ for $30 \mathrm{~min}$ at $4^{\circ} \mathrm{C}$. A 25:24:1 (v/v/v) equal volume of neutral phenol: chloroform: isoamyl alcohol were used for the extraction of the DNA from the supernatant. Then, electrophoretic analysis was performed on $1.5 \%$ agarose gels containing $0.1 \mu \mathrm{g} / \mathrm{ml}$ ethidium bromide $(\mathrm{EtBr})$.

Flow cytometry analysis for cell cycle analysis and apoptosis. For the measurement of the sub-G1 phase, the cells treated with morin were collected, washed with cold PBS, and centrifuged. The pellet was fixed in $75 \%(\mathrm{v} / \mathrm{v})$ ethanol for $1 \mathrm{~h}$ at $4^{\circ} \mathrm{C}$. The cells were washed once with PBS and resuspended in cold PI solution $(50 \mu \mathrm{g} / \mathrm{ml})$ containing RNase A $(0.1 \mathrm{mg} /$ $\mathrm{ml}$ ) in PBS (pH 7.4) for $30 \mathrm{~min}$ in the dark. The Annexin V double staining was performed using $5 \mu \mathrm{l}$ of the Annexin V conjugate which was added to each $100 \mu$ l of cell suspension for $15 \mathrm{~min}$, followed by adding $400 \mu \mathrm{l}$ of Annexin V-binding buffer and mixed gently. Then the samples were placed on ice. Flow cytometry analyses were performed with Beckman coulter cytomics FC 500 (Becton Dickinson, San Jose, CA, USA). The sub-G1 population was calculated to estimate the apoptotic cell population.

Measurement of mitochondrial membrane potential (MMP, $\left.\Delta \Psi_{m}\right)$ and reactive oxygen species (ROS) generation. The $\operatorname{MMP}\left(\Delta \Psi_{\mathrm{m}}\right)$ in living cells were measured by flow cytometry with the lipophilic cationic probe JC-1, a ratiometric, dualemission fluorescent dye. There are two excitation wavelengths, $527 \mathrm{~nm}$ (green) for the monomer form and $590 \mathrm{~nm}$ (red) for the J-aggregate form. Quantitation of green fluorescent signals reflects the amount of damaged mitochondria. The cell were harvested and re-suspended in $500 \mu \mathrm{l}$ of PBS, incubated with $10 \mu \mathrm{M} \mathrm{JC}-1$ for $20 \mathrm{~min}$ at $37^{\circ} \mathrm{C}$. For ROS measurement, the cells were incubated with $10 \mu \mathrm{M} 2^{\prime}, 7^{\prime}$-dichlorofluorescein diacetate (DCF-DA) at $37^{\circ} \mathrm{C}$ for $30 \mathrm{~min}$. The cells were then washed with ice-cold PBS and harvested. Fluorescence was determined by a FACS flow cytometer.

Western blot analysis. The extracted proteins were quantified using the Bio-Rad protein assay (Bio-Rad Laboratories, Inc., Hercules, CA, USA). For the mitochondrial fraction, the Mitochondria Isolation kit for cultured cells (Thermo Fisher Scientific) was used and the protocol was followed as per the manufacturer's instructions. The final supernant was a cytosol fraction, and the pellet contained the isolated mitochondria. The proteins of the extracts were resolved by electrophoresis, electrotransferred to a polyvinylidene difluoride membrane (Millipore, Bedford, MA, USA). The membrane was then incubated with the primary antibodies followed by a conjugated secondary antibody to peroxidase. An ECL detection system was used to visualize the developed blots.

In vitro caspase activity assay. Caspase activity was measured using colorimetric assay kits, which contained the following synthetic tetrapeptides, labeled with p-nitroaniline (pNA): Asp-Glu-Val-Asp (DEAD) for caspase-3, Ile-Glu-Thr-Asp (IETD) for caspase-8 and Leu-Glu-His-Asp (LEHD) for caspase-9. The cells were lysed using the lysis buffer provided in the kit. The supernatants were collected and incubated with the supplied reaction buffer containing dithiothreitol and 


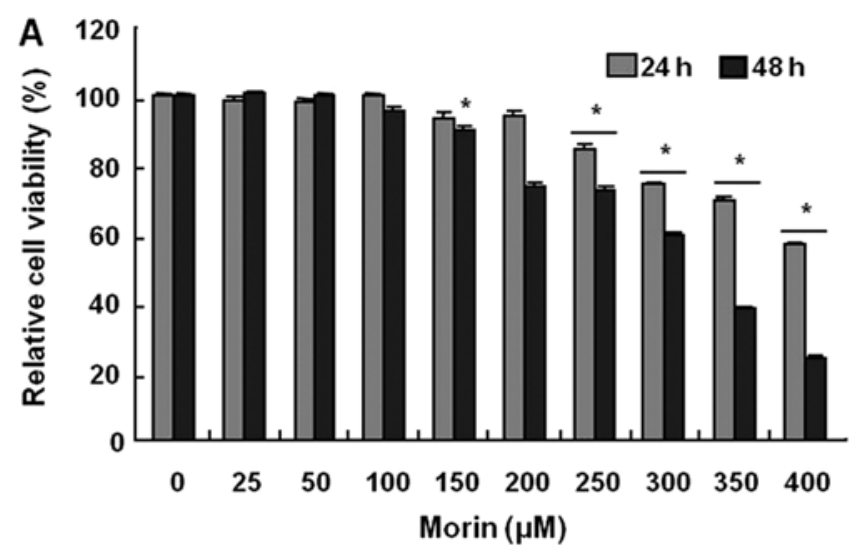

B $\quad \operatorname{Morin}(\mu \mathrm{M})$

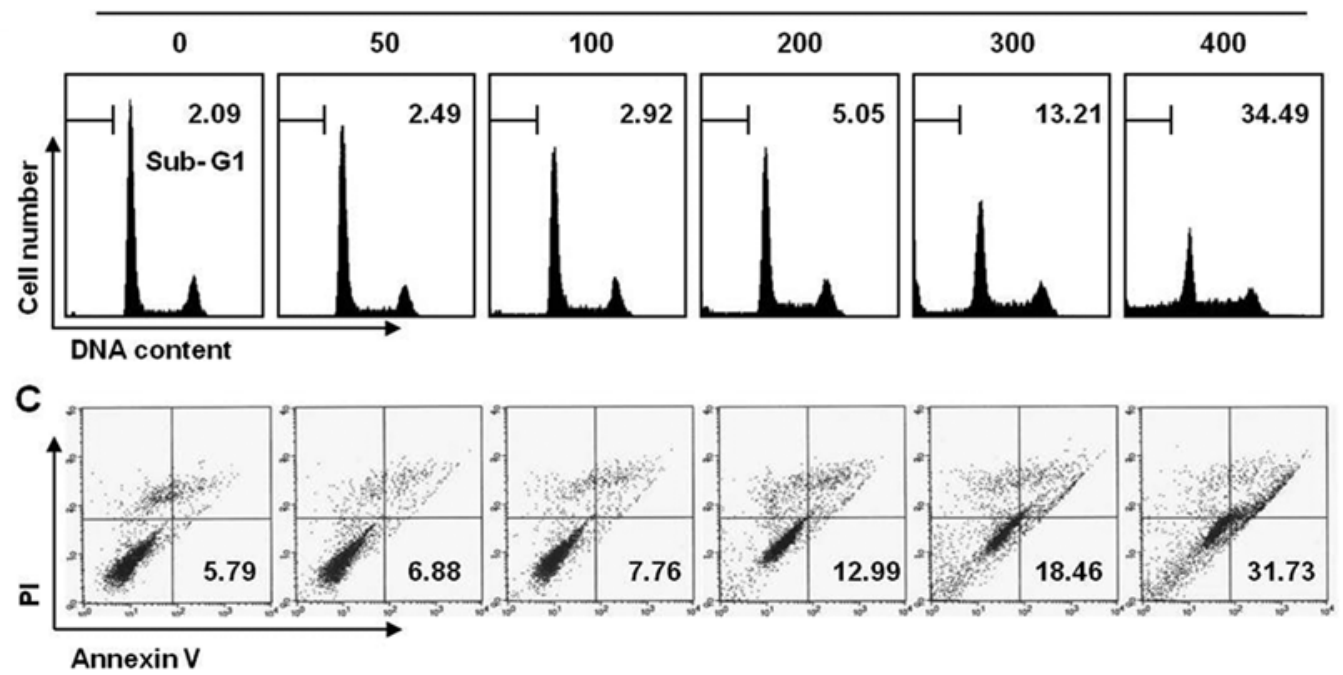

Figure 1. Effects of morin on proliferation of HCT-116 human colon cancer cells and apoptosis induction. The growth inhibition and cytotoxicity of morin are dose- and time-dependent. HCT- 116 cells were seeded at the density of $5 \times 10^{4}$ cells per ml. The cells were treated with indicated concentrations of morin for $48 \mathrm{~h}$. (A) Cell viability was analyzed by MTT assay. The data are shown as means \pm SD of three independent experiments. ${ }^{*} \mathrm{P}<0.05$ between the treated and the untreated control group. (B) Cell cycle analysis to quantify the degree of morin-induced apoptosis, and to determine whether morin induces cell cycle arrest. Sub-G1 DNA content represents the fractions undergoing apoptotic DNA degradation by morin treatment. (C) Flow cytomery for the dual staining of Annexin V and PI. Annexin V + PI- cells indicate the cells undergoing early apoptosis.

substrates at $37^{\circ} \mathrm{C}$. The caspase activity was determined by absorbance at $405 \mathrm{~nm}$ on the microplate reader.

Statistics. Each experiment was performed in triplicate. The results are expressed as means \pm SD. Significant differences were determined using the one-way analysis of variance (ANOVA) with post-test Neuman-Keuls in the cases at least three treatment groups and Student's t-test for two group comparison. Statistical significance was defined as $\mathrm{P}<0.05$.

\section{Results}

Effects of morin on proliferation of HCT-116 human colon cancer cells and apoptosis induction. To investigate the antitumor activity, HCT-116 cells were treated with indicated concentrations of morin $(\leq 400 \mu \mathrm{g} / \mathrm{ml})$ for $48 \mathrm{~h}$. The growth of HCT-116 cells were inhibited by morin treatment in a dosedependent manner, The $\mathrm{IC}_{50}$ obtained on $48 \mathrm{~h}$-morin treatment was less than $350 \mu \mathrm{g} / \mathrm{ml}$ (Fig. 1A). Next, we performed cell cycle analysis to assess the sub-G1 DNA population and also to study the involvement of morin in inducing cell cycle arrest. As shown in Fig. 1B, morin induced significant accumulation of cells with sub-G1 DNA content (apoptotic cell population) and substantially decreased the G1 fractions; in contrast, the and S phase and G2M population displayed a modest expansion. Lastly, we measured the early apoptotic cells (Annexin $\mathrm{V}^{+} / \mathrm{PI}^{-}$) by flow cytometry and observed a dose-dependent increase in the early apoptotic cells (Fig. 1C). These results suggest that morin induces apoptosis in HCT 116 human colon cancer cells.

Morin-induced cell death is associated with caspase activation. Caspases are the principal key mediators in inducing apoptosis and they contribute by leading apoptotic cell death to irreversible cell death (9). Next, we performed western blot analyses, to assess the expression of caspases and substrates (PARP). Morin decreased the expression levels of procaspase-3, procaspase- 8 , and procaspase- 9 , which indicated caspase activation. The induction of the cleavage of procaspase- 3 and procaspase- 3 were prominent in the cells treated 
A

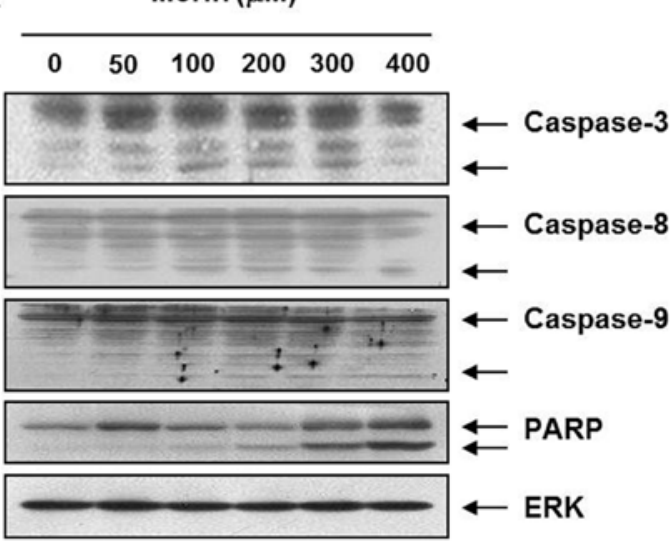

B

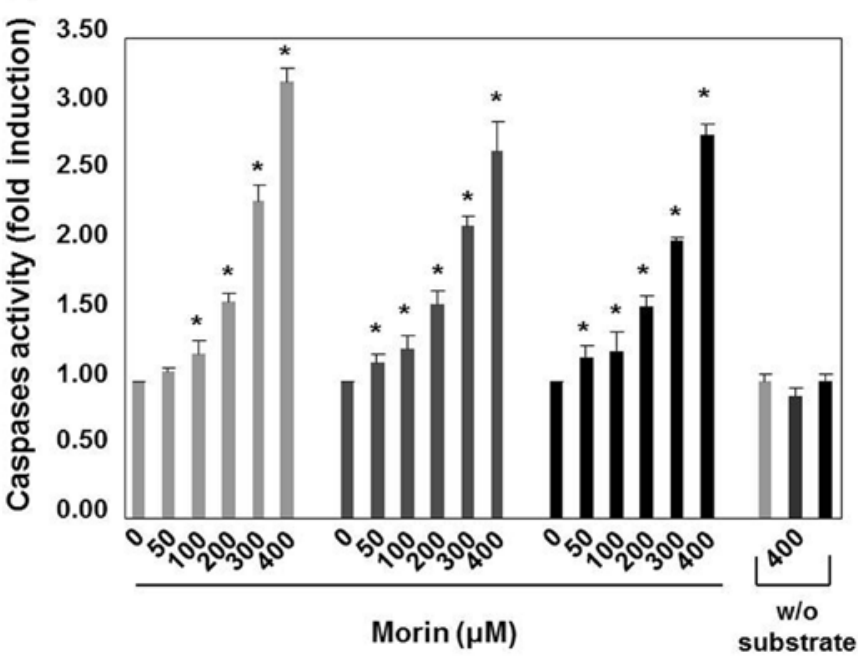

Figure 2. Morin-induced cell death is associated with caspase activation in HCT-116 cells. (A) Western blot analysis for the effects of morin on the caspase activation and PARP cleavage. Morin activates caspases and induces the cleavage of PARP in a dose-dependent manner. HCT-116 cells were incubated at indicated concentrations of morin for $48 \mathrm{~h}$. The membranes were probed with the anti-caspase- 3 , anti-caspase- 8 , anti-caspase- 9 and antiPARP antibodies. (B) The cell lysates from the cells treated with morin for $48 \mathrm{~h}$ were assayed for in vitro caspase- $3,-8$ and -9 activity using DEVD-pNA, IETD-pNA and LEHD-pNA, respectively, as substrates. The released fluorescent products were measured. Data are expressed as mean \pm SD of three independent experiments. ${ }^{*} \mathrm{P}<0.05$ vs. untreated control.

with morin for $48 \mathrm{~h}$ (Fig. 2A). Morin also induced the cleavage of PARP (Fig. 2A). Next, we confirmed morin-induced activation of caspases-3, -8 and -9 with caspase activity assay, which revealed that caspase $-3,-8$ and -9 activation increased in a dose-dependent manner (Fig. 2B). These findings suggest that morin may induce apoptosis through both the intrinsic and the extrinsic pathways.

Morin upregulates Fas receptors that are associated with the death receptor-mediated apoptosis. To determine which apoptotic pathway is involved in the morin-induced apoptosis; we measured the expression of TRAIL receptors (DR4, DR5), TRAIL, Fas receptor (Fas), and Fas ligand (FasL). Western blot analysis revealed that Fas receptor is upregulated by morin in a dose-dependent manner (Fig. 3), suggesting that morin induces the death receptor-mediated apoptosis through the extrinsic pathway.

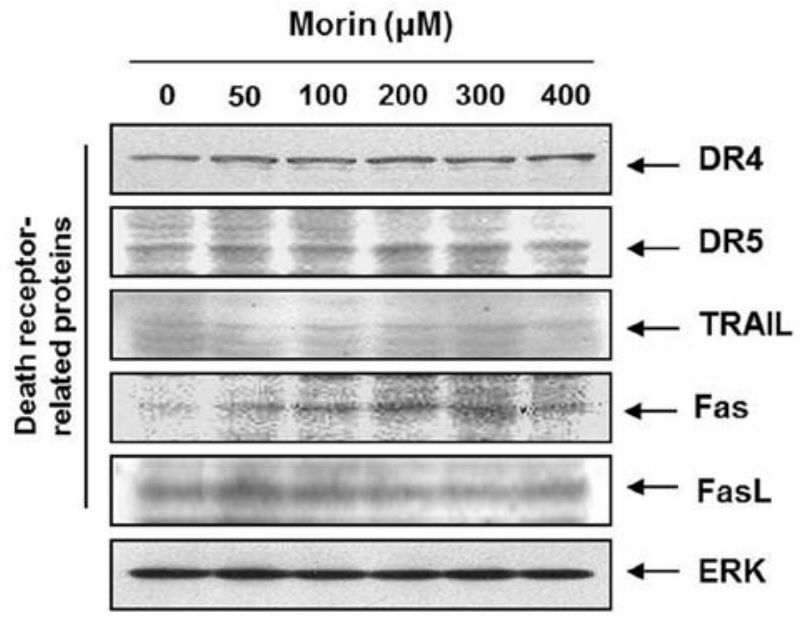

Figure 3. The effects of morin on activation of death receptor pathway that is associated with caspase- 8 activation in HCT-116 cells. Western blot analysis for the effects of morin on the TNF-related apoptosis-inducing ligand (TRAIL) receptor DR5 pathway. The cells $\left(5 \times 10^{4}\right)$ were incubated at the indicated concentrations of morin for $48 \mathrm{~h}$. The results are from one representative of two independent experiments that showed similar patterns.

Morin-induced apoptosis is associated with loss of MMP $\left(\Delta \Psi_{m}\right)$, generation of reactive oxygen species $(R O S)$, but NAC does not block ROS production. Mitochondria play a central role in apoptosis and mitochondrial depolarization occurs as an early event of apoptosis (9). We measured the changes in MMP $\left(\Delta \Psi_{\mathrm{m}}\right)$ after morin treatment. As shown in Fig. 4A, morin began to induce loss of $\operatorname{MMP}\left(\Delta \Psi_{\mathrm{m}}\right)$ at the low concentration of $50 \mu \mathrm{g} / \mathrm{ml}$. This result suggested that morin-induced apoptosis may be associated with mitochondrial depolarization. As ROS generation is one of the popular mechanisms for mitochondria-related apoptosis, a clear understanding is required as to whether intracellular ROS generation was contributing to the mitochondrial depolarization in morintreated cells $(9,10)$. We measured ROS production $6 \mathrm{~h}$ after morin treatment. As shown in (Fig. 4B), morin induced ROS production, but the ROS production was not reduced by the ROS scavenger, N-acetyl-L-cysteine (NAC). To confirm this finding, we further assessed the influence of N-acetylL-cysteine (NAC) on morin-induced cell death. MTT, DNA fragmentation test, and flow cytometry for early apoptotic cell detection (Annexin $\mathrm{V}^{+} / \mathrm{PI}^{-}$) also suggested that ROS generation did not play an important role in cell death (Fig. 4C-E). These results suggest that morin may induce ROS generation, but that NAC could not prevent either morin-induced ROS generation or apoptosis.

Modulation of Bcl-2 and IAP family proteins by morin in $H C T-116$ cells. Bcl-2 family members serve as determinants of apoptotic cell death through maintaining the $\operatorname{MMP}\left(\Delta \Psi_{\mathrm{m}}\right)$. In response to apoptotic signaling, Bid interacts with another Bcl-2 family protein (anti-apoptotic proteins), and involves in the opening of mitochondrial voltage-dependent anion channel (VDAC). Thus, the opened channel results in the release of cytochrome $\mathrm{c}$ and other pro-apoptotic factors from the mitochondria, leading to activation of caspases. To elucidate further underlying mechanisms of the mitochondrial pathway-related apoptosis induced by morin, we assessed the levels of Bcl-2 
A

Morin $(\mu \mathrm{M})$

\begin{tabular}{llllll}
\hline 0 & 50 & 100 & 200 & 300 & 400
\end{tabular}

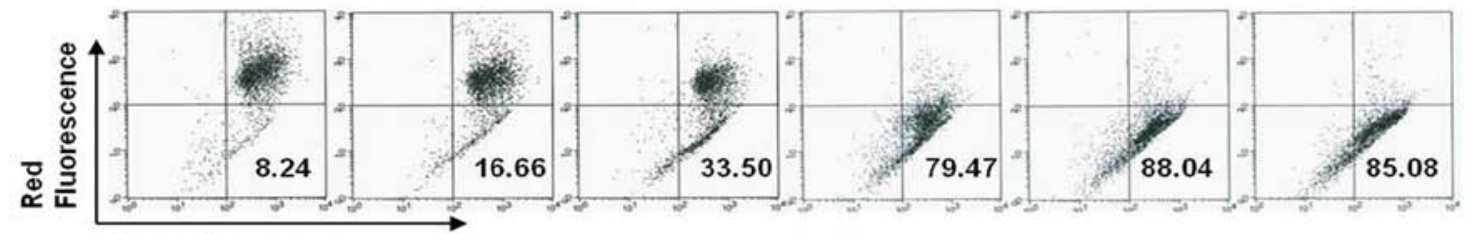

Green Fluorescence

B

Time (h)
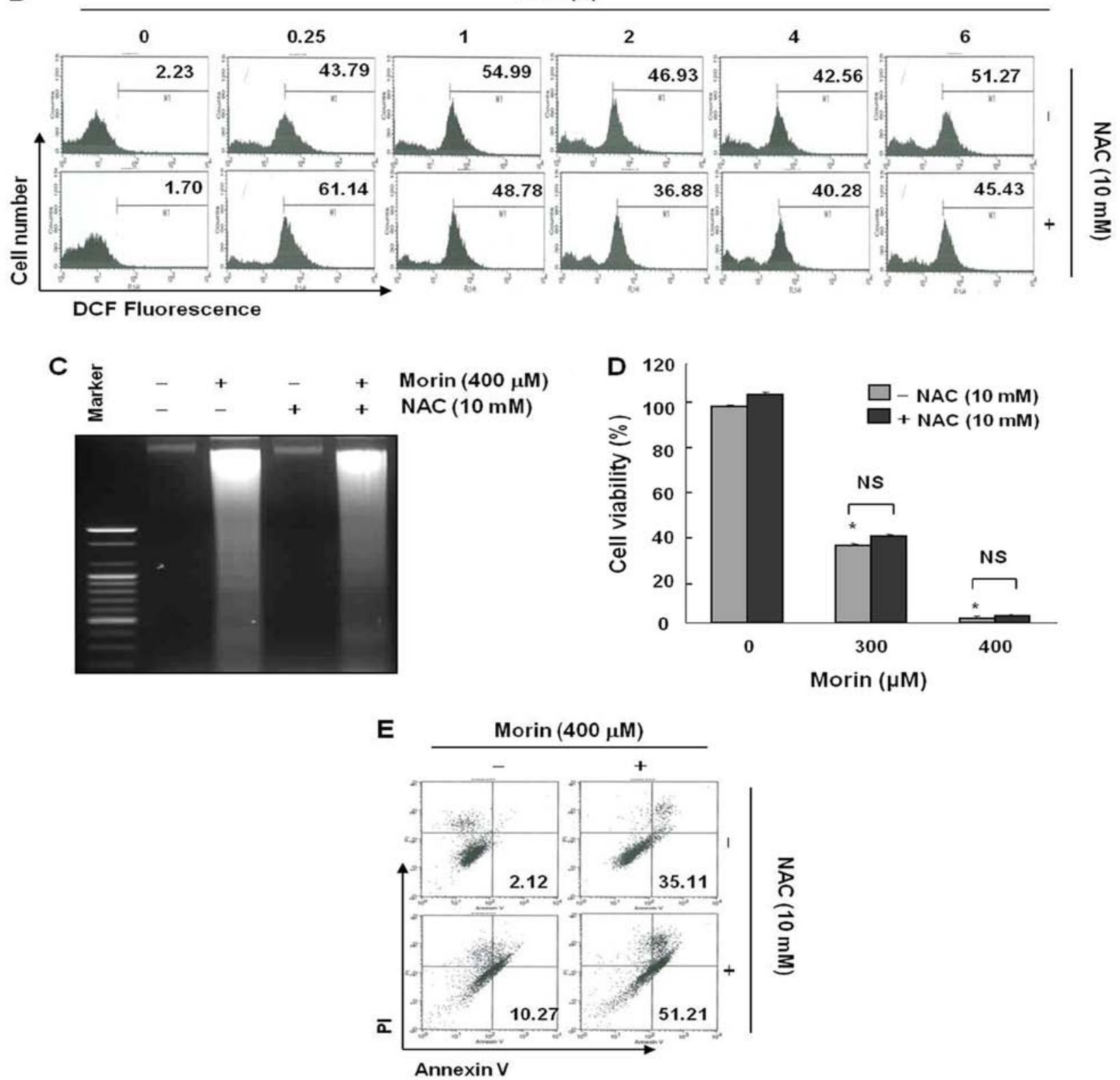

Figure 4. Role of mitochondria and ROS in morin-induced apoptosis in HCT-116 cells. (A) Morin induced loss of MMP $\left(\Delta \Psi_{\mathrm{m}}\right)$ in a dose-dependent manner. The cells were stained with JC-1 and incubated at $37^{\circ} \mathrm{C}$ for $30 \mathrm{~min}$. The mean JC-1 fluorescence intensity was assessed by a flow cytometer. (B) For the assessment of ROS levels, the cells were incubated with $10 \mu \mathrm{M} 2^{\prime}, 7^{\prime}$-dichlorofluorescein diacetate (DCF-DA) for 30 min after morin treatment. N-acetyl-L-cysteine (NAC) $(0.5 \mathrm{mM})$ treatment was given $1 \mathrm{~h}$ before morin treatment. Fragmented nuclei were observed in the morin-treated group under a fluorescent microscope. (C) DNA fragmentation test. (D) Cell cycle analysis for Sub-G1 DNA content that represents the fractions undergoing apoptosis. (E) Flow cytomery for the dual staining of Annexin V and PI. Annexin $\mathrm{V}^{+} / \mathrm{PI}^{-}$cells indicate the cells undergoing early apoptosis. The proportion is expressed as a percentage.

family members. Western blotting revealed that morin induced Bid activation and Bax upregulation while Bcl-2 expression was reduced (Fig. 5A). Bid is the substrate of caspase-8 (11). This finding also supports the above finding in Fig. 4. Next 


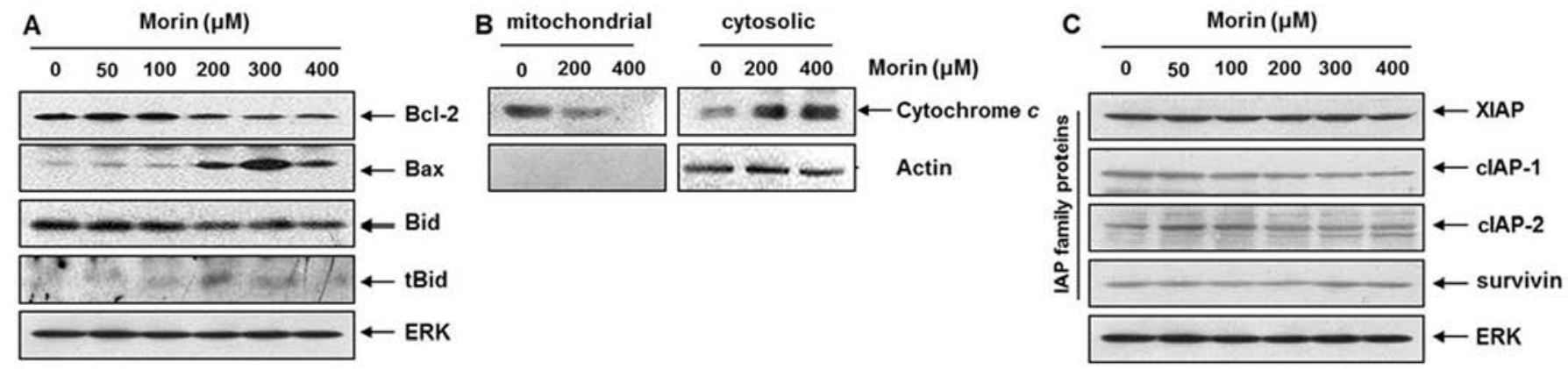

Figure 5. Modulation of Bcl-2 and IAP family proteins during morin-induced apoptosis. Western blot analysis for the effects of morin on Bcl-2 and IAP family members. The cells $\left(5 \times 10^{4}\right)$ were incubated at the indicated concentrations for $48 \mathrm{~h}$. (A) Morin suppressed the expression of Bcl-2, and activated Bid and BAX proteins. (B) Cytochrome $\mathrm{c}$ was released from mitochondria (C) Morin slightly downregulated cIAP1 in a dose-dependent manner. The results are from one representative of two independent experiments that showed similar patterns.

A

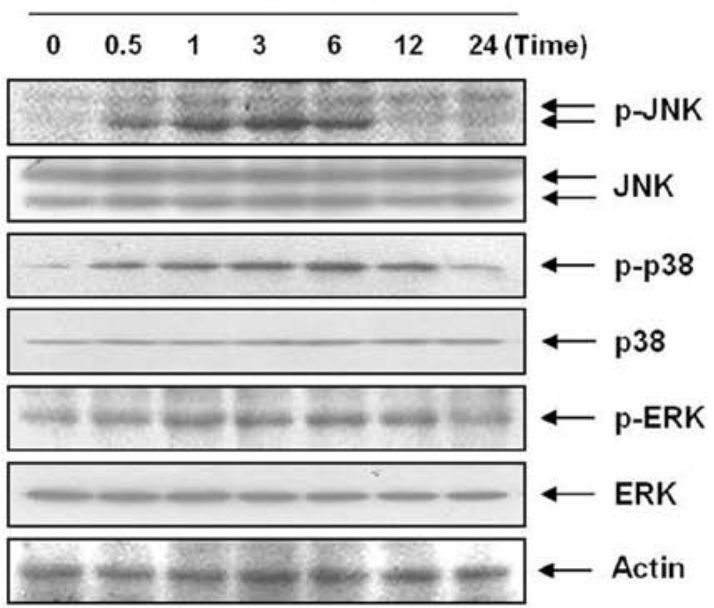

B

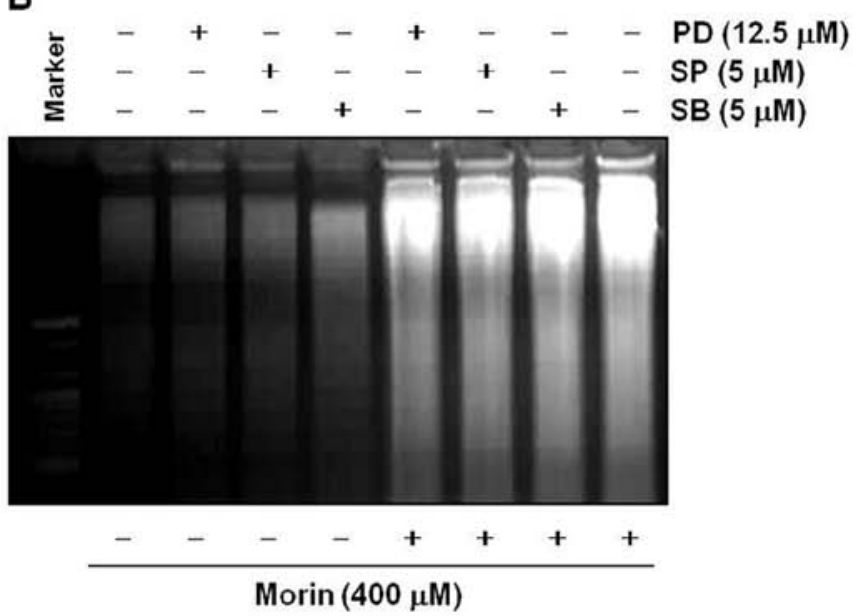

C

UT
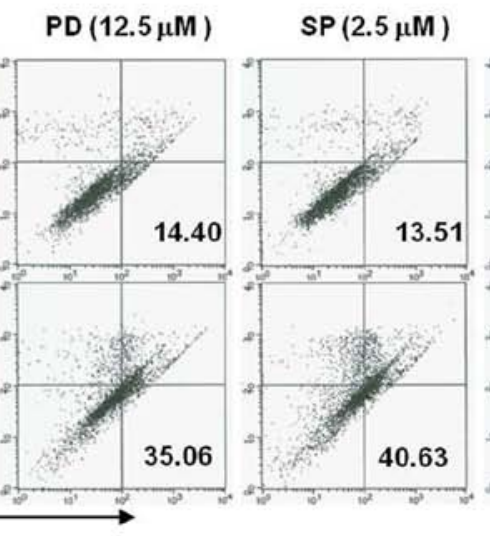

$\mathrm{SB}(10 \mu \mathrm{M})$

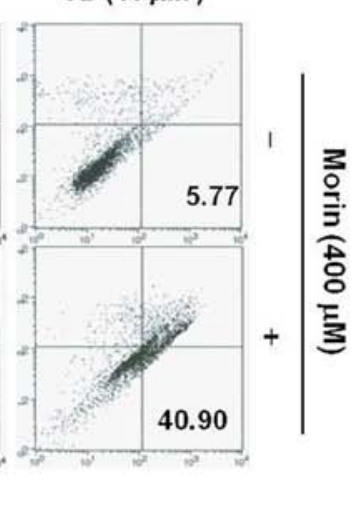

Figure 6. Role of MAPK in morin-induced apoptosis in HCT-116 cells. The cells $\left(5 \times 10^{4}\right)$ were incubated at indicated concentrations for $48 \mathrm{~h}$. (A) Western blot analysis for the effects of morin on MAPK. Morin activated MAPK phosphorylation. Whole-cell extracts were prepared, and western blotting was performed with the indicated antibodies. (B and C) Apoptotic effects of morin were assessed by (B) DNA fragmentation test and (C) flow cytomery for the dual staining of Annexin V. The cells were treated with MAPK inhibitors (the JNK inhibitor SP600125, SP, the P38 inhibitor SB203580, SB, and the ERK inhibitor PD98059, PD).

we confirmed that morin induced cytochrome c release from mitochondria (Fig. 5B). Further, we tested the expression of inhibitor of apoptosis protein (IAP) family members which also play a key role in caspase-dependent apoptosis. Western blotting revealed that morin mildly suppressed cIAP1, but did influence other IAP family members (Fig. 5C). These finding indicated that morin-induced apoptosis was associated with modulation of Bcl-2, Bid and cIAP1 proteins in HCT-116 cells, suggesting mitochondrial pathway is also important in morininduced apoptosis.

Morin-induced apoptosis is associated with suppression of Akt pathway in HCT-116 cells. Mitogen-activated protein kinase (MAPK) is involved in cell proliferation, survival, 
A

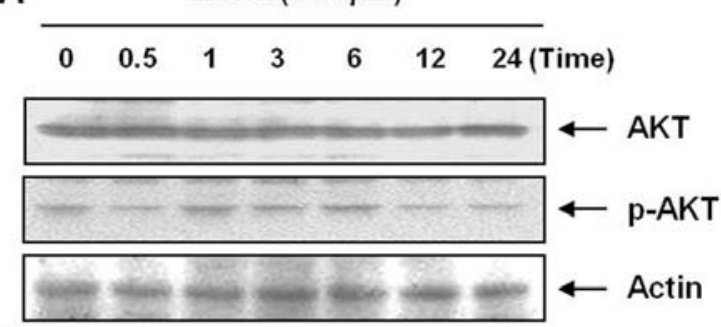

B

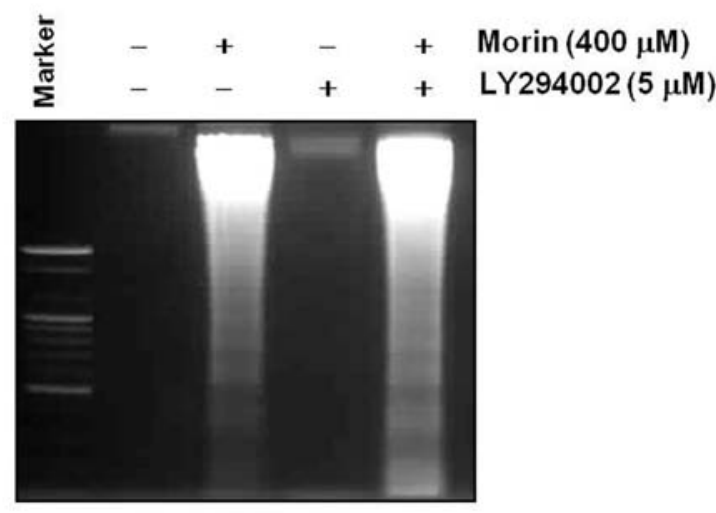

C

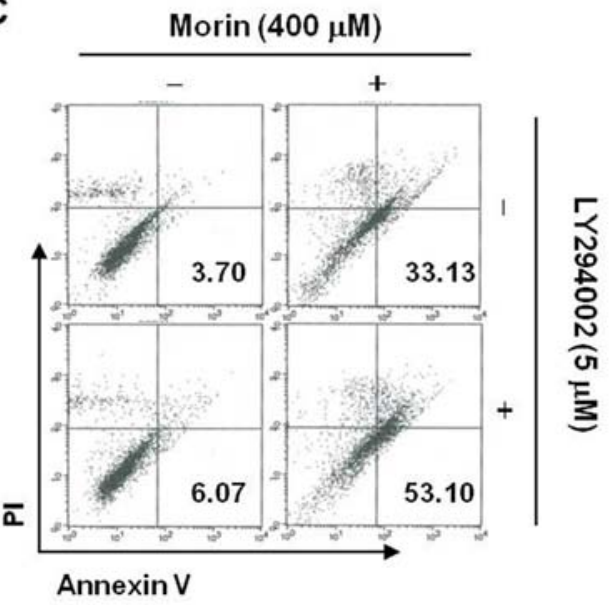

Figure 7. Role of Akt in morin-induced apoptosis in HCT-116 cells. The cells $\left(5 \times 10^{4}\right)$ were incubated at indicated concentrations for $48 \mathrm{~h}$. (A) Western blot analysis for the effects of morin on Akt activity. Morin suppressed Akt phosphorylation. Whole-cell extracts were prepared, and western blot was performed with the indicated antibodies. (B and C) Apoptotic effects of morin were assessed by (B) DNA fragmentation test and (C) flow cytometry for the dual staining of Annexin V. The cells were treated with the Akt inhibitor LY294002.

and apoptosis (12). To understand the mechanism involved in morin-induced apoptosis, we first studied the changes in MAPK activation after morin treatment. Western blot analysis showed that morin began to induce phophorylation of JNK, p38-MAPK and ERK at $1 \mathrm{~h}$ after the treatment (Fig. 6A). To confirm the involvement of the MAPK in morin-induced apoptosis, we assessed the changes in the population of apoptotic cell death after the MAPK inhibitors. However, DNA fragmentation test and flow cytometry assay for Annexin $\mathrm{V}^{+} / \mathrm{PI}^{-}$cells revealed that the inhibitors of MAPK only slightly influenced morin-induced apoptosis (Fig. 6B and C). These data suggested that of MAP kinases were activated by morin treatment, but the activation of MAP kinases was not associated with morin-induced apoptosis. As is well known, Akt/PI3k pathway is also important in cell survival, therefore we assessed the changes in Akt activation after morin treatment (13). Western blot analysis showed that morin began to suppress phophorylation of Akt at $12 \mathrm{~h}$ after the treatment (Fig. 7A). To confirm the involvement of Akt in morin-induced apoptosis, we assessed the changes in the population of apoptotic cell death after the inhibitor of Akt. In contrast to the MAPK results, DNA fragmentation test and flow cytometry assay for Annexin $\mathrm{V}^{+} / \mathrm{PI}^{-}$cells revealed that a small dose of the Akt inhibitor LY294002 augmented the morin-induced apoptosis (Fig. 7B and C). These findings suggested that morin induces apoptosis at least in part by suppression of Akt activity.

\section{Discussion}

This study determined whether morin has anticancer properties in human cancer cells and further investigated the underlying mechanisms involved in its anticancer effects. We found that morin induced caspase-dependent apoptosis in a dose-dependent manner. The induction of apoptosis was triggered through both the extrinsic and the intrinsic pathway by modulating Fas receptor and Bcl-2 family members. The modulation of these proteins was related to suppression of Akt activity. There are substantial evidence reporting that apoptosis (type I programmed cell death) is the principal underlying mechanism through which various anticancer and chemo-preventive agents, including natural compounds, exert anticancer effects (14). Apoptosis is initiated by the activation of a set of death effector cysteine proteases called caspases. In most of the apoptotic processes, caspase- 8 is involved in the extrinsic pathway, caspase-9 is involved in the intrinsic pathway, and caspase-3 plays a pivotal role in the terminal and execution phase of apoptosis (15). This study demonstrated that morin induced caspase- $8,-9$, and -3 activation and the subsequent cleavages of PARP $(89 \mathrm{kDa})$.

Caspase- 8 can be triggered through either the intrinsic or the extrinsic pathway (16). However, the early caspase induction depends on the extrinsic pathway. As shown in Fig. 2, the activation of caspase- 3 and -8 occurs at a lower concentration than caspase-9 activation. This finding also supports that morin-induced apoptosis is associated with the extrinsic pathway activation. To confirm this finding, we assessed the apoptotic pathways and found that morin induced apoptosis by upregulating Fas receptor and thereby activating the Bid protein, which is a natural substrate of caspase-8 (11). This finding agrees with the results of caspase activation by morin (Fig. 2). Although the finding that morin upregulates Fas receptor expression has not been reported yet, other flavonoids have already been reported to induce apoptosis by upregulating Fas receptor or TRAIL receptor (17). Also, the modulation of Bcl-2 family members and Fas receptors is associated with suppression of Akt activity $(18,19)$.

ROS generation is one of the important mechanisms in induction of apoptosis particularly relating to both deathreceptors and mitochondrial pathway (20). Therefore, for the evaluation of their underlying mechanisms, we assessed ROS generation in the morin-treated cells. We found that morin induced ROS generation. However, NAC could not reverse morin-induced ROS generation and apoptosis although, in 


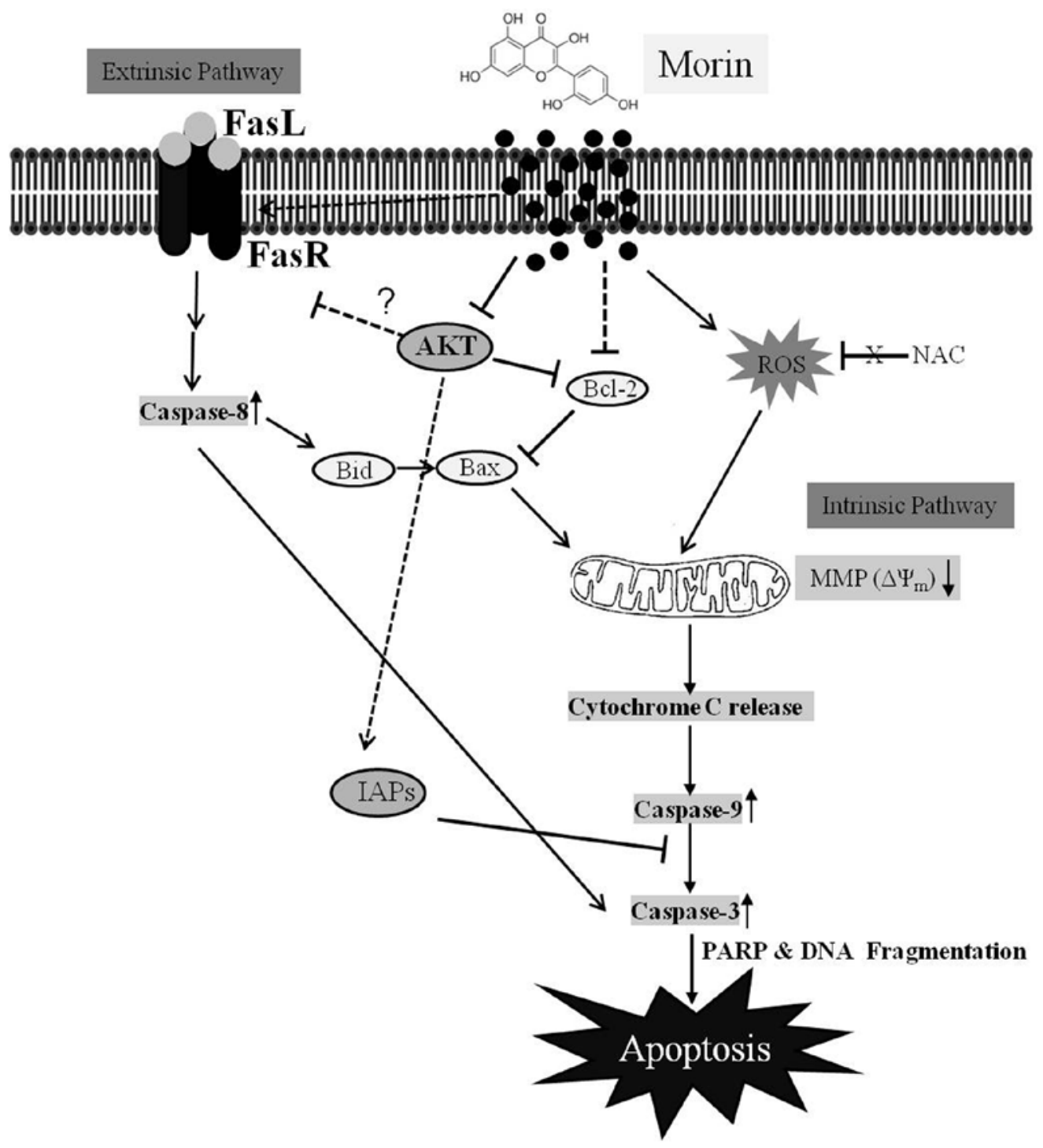

Figure 8. Schematic representation on apoptotic effects of morin on HCT-116 human colon cancer cells. Morin induces death receptor-mediated apoptosis by upregulating Fas receptor, and then activates caspase- 8 . The activated caspase- 8 activates Bid, and thus the Bid induces loss of mitochondrial membrane potential (MMP, $\Delta \Psi_{\mathrm{m}}$ ) through activation of Bax. Morin induced ROS generation which was not blocked by N-acetylcysteine. Morin also suppressed Bcl-2 and cIAP-1, which contribute to augmenting morin-induced caspase-9-mediated apoptosis. These findings indicated that the intrinsic pathway also plays an important role in morin-induced apoptosis. The suppressed Akt activity by morin may contribute to the suppression of the expression of Bcl-2 and cIAP-1. Morin induces caspase-dependent apoptosis through the extrinsic pathway by upregulating Fas receptor as well as through the intrinsic pathway by modulating Bcl-2 and IAP family members, and Akt is the critical upstream signaling that regulates the apoptotic effect of morin in human colon cancer HCT-116 cells. $(\rightarrow$ indicates activation, $\perp$ indicates inhibition, --- indicates indirect or multiple pathways).

some of death receptor-mediated apoptosis, ROS generation can be blocked by NAC. In this study, we could not investigate the detained mechanisms as to why NAC did not reverse morin-induced ROS generation and apoptosis. There is a possibility that NAC behaves as apoptosis inducer because NAC in certain conditions is able to induce apoptosis $(21,22)$.

In the process of apoptosis, Bcl-2 and IAP family members play significant roles $(9,23)$. We observed that morin suppressed anti-apoptotic proteins Bcl-2, and cIAP-1. Hence, we conclude that morin induce apoptosis at least in part through mitochondrial pathway We also suggest an association between morin-induced apoptosis and Akt inhibition. Akt is well known to play a crucial role in triggering apoptosis by regulating Bcl-2 and IAP family members $(24,25)$. In addition, we demonstrated that morin inhibited Akt activity, and the combination therapy of morin with the Akt inhibitor LY294002 showed an additive effect. These findings support that morin induced apoptosis at least in part through inhibition of Akt activity. In addition, a previous study demonstrated that morin could inhibit PI3/Akt with docking analysis between PI3K and morin (25).

The drawback of this study is that we used the maximum concentration that is 2- to 5-fold higher than used in previous studies showing antitumor effect of morin. Thus, the concentration used in performing this study is one of the obstacles for pursuing in vivo experiments. However, we previously found that morin hardly shows any anticancer effects by MTT assay (26). In that study, morin did not show any apoptotic effects up to $200 \mu \mathrm{M}$, but it showed clear anticancer effects in vivo at daily dosing with $50 \mathrm{mg} / \mathrm{kg}$ for 7 days without 
showing any toxicity. This result support that morin is a safe natural product that can show anticancer effects in vivo.

In summary, this study demonstrated that morin suppressed cell viability and induced caspase-dependent apoptosis in HCT-116 cells (Fig. 8). Apoptosis induced by morin was triggered through the intrinsic and extrinsic pathways by modulating Bcl-2 and IAP family members, and FAS receptor expression. The suppression of Akt phosphorylation was involved in morin-induced apoptosis in HCT-116 cells. This study provides substantial evidence that morin may have anticancer properties in colon cancer cells.

\section{Acknowledgements}

This study was supported by grants from the National R\&D Program for Cancer Control, Ministry of Health and Welfare, Republic of Korea (0820050).

\section{References}

1. Jung KW, Won YJ, Kong HJ, Oh CM, Seo HG and Lee JS Prediction of cancer incidence and mortality in Korea, 2013. Cancer Res Treat 45: 15-21, 2013.

2. Jung KW, Won YJ, Kong HJ, Oh CM, Lee DH and Lee JS: Cancer statistics in Korea: Incidence, mortality, survival, and prevalence in 2011. Cancer Res Treat 46: 109-123, 2014.

3. Lee JE, Männistö S, Spiegelman D, Hunter DJ, Bernstein L, van den Brandt PA, Buring JE, Cho E, English DR, Flood A, et al: Intakes of fruit, vegetables, and carotenoids and renal cell cancer risk: A pooled analysis of 13 prospective studies. Cancer Epidemiol Biomarkers Prev 18: 1730-1739, 2009.

4. Gandini S, Merzenich H, Robertson C and Boyle P: Metaanalysis of studies on breast cancer risk and diet: The role of fruit and vegetable consumption and the intake of associated micronutrients. Eur J Cancer 36: 636-646, 2000.

5. Hatcher H, Planalp R, Cho J, Torti FM and Torti SV: Curcumin: From ancient medicine to current clinical trials. Cell Mol Life Sci 65: 1631-1652, 2008.

6. Kawabata K, Tanaka T, Honjo S, Kakumoto M, Hara A, Makita H, Tatematsu N, Ushida J, Tsuda $\mathrm{H}$ and Mori H: Chemopreventive effect of dietary flavonoid morin on chemically induced rat tongue carcinogenesis. Int J Cancer 83: 381-386, 1999.

7. Brown J, O'Prey J and Harrison PR: Enhanced sensitivity of human oral tumours to the flavonol, morin, during cancer progression: Involvement of the Akt and stress kinase pathways. Carcinogenesis 24: 171-177, 2003.

8. Kerr JF, Wyllie AH and Currie AR: Apoptosis: A basic biological phenomenon with wide-ranging implications in tissue kinetics. Br J Cancer 26: 239-257, 1972.

9. Hengartner MO: The biochemistry of apoptosis. Nature 407: 770-776, 2000

10. Miyajima A, Nakashima J, Yoshioka K, Tachibana M, Tazaki H and Murai M: Role of reactive oxygen species in cis-dichlorodiammineplatinum-induced cytotoxicity on bladder cancer cells Br J Cancer 76: 206-210, 1997.
11. Timmer JC and Salvesen GS: Caspase substrates. Cell Death Differ 14: 66-72, 2007.

12. Pearson G, Robinson F, Beers Gibson T, Xu BE, Karandikar M, Berman K and Cobb MH: Mitogen-activated protein (MAP) kinase pathways: Regulation and physiological functions. Endocr Rev 22: 153-183, 2001.

13. Qian J, Zou Y, Rahman JS, Lu B and Massion PP: Synergy between phosphatidylinositol 3-kinase/Akt pathway and Bcl-xL in the control of apoptosis in adenocarcinoma cells of the lung. Mol Cancer Ther 8: 101-109, 2009.

14. Chun KH, Kosmeder JW II, Sun S, Pezzuto JM, Lotan R, Hong WK and Lee HY: Effects of deguelin on the phosphatidylinositol 3-kinase/Akt pathway and apoptosis in premalignant human bronchial epithelial cells. J Natl Cancer Inst 95: 291-302, 2003.

15. Thornberry NA and Lazebnik Y: Caspases: Enemies within. Science 281: 1312-1316, 1998.

16. Ashkenazi A: Targeting death and decoy receptors of the tumour-necrosis factor superfamily. Nat Rev Cancer 2: 420-430, 2002.

17. Jin CY, Park C, Cheong J, Choi BT, Lee TH, Lee JD, Lee WH, Kim GY, Ryu CH and Choi YH: Genistein sensitizes TRAILresistant human gastric adenocarcinoma AGS cells through activation of caspase-3. Cancer Lett 257: 56-64, 2007.

18. Nyåkern M, Cappellini A, Mantovani I and Martelli AM: Synergistic induction of apoptosis in human leukemia T cells by the Akt inhibitor perifosine and etoposide through activation of intrinsic and Fas-mediated extrinsic cell death pathways. Mol Cancer Ther 5: 1559-1570, 2006.

19. Han MH, Lee WS, Jung JH, Jeong JH, Park C, Kim HJ, Kim G, Jung JM, Kwon TK, Kim GY, et al: Polyphenols isolated from Allium cepa $\mathrm{L}$. induces apoptosis by suppressing IAP-1 through inhibiting PI3K/Akt signaling pathways in human leukemic cells. Food Chem Toxicol 62: 382-389, 2013.

20. Simon HU, Haj-Yehia A and Levi-Schaffer F: Role of reactive oxygen species (ROS) in apoptosis induction. Apoptosis 5: 415-418, 2000.

21. Zafarullah M, Li WQ, Sylvester J and Ahmad M: Molecular mechanisms of $\mathrm{N}$-acetylcysteine actions. Cell Mol Life Sci 60: 6-20,2003.

22. Wang AL, Wang JP, Wang H, Chen YH, Zhao L, Wang LS, Wei W and Xu DX: A dual effect of $\mathrm{N}$-acetylcysteine on acute ethanol-induced liver damage in mice. Hepatol Res 34: 199-206, 2006.

23. Deveraux QL and Reed JC: IAP family proteins - suppressors of apoptosis. Genes Dev 13: 239-252, 1999.

24. Franke TF, Hornik CP, Segev L, Shostak GA and Sugimoto C: PI3K/Akt and apoptosis: Size matters. Oncogene 22: 8983-8998, 2003.

25. Sivaramakrishnan V and Devaraj SN: Morin fosters apoptosis in experimental hepatocellular carcinogenesis model. Chem Biol Interact 183: 284-292, 2010.

26. Jin H, Lee WS, Eun SY, Jung JH, Park HS, Kim G, Choi YH, Ryu CH, Jung JM, Hong SC, et al: Morin, a flavonoid from Moraceae, suppresses growth and invasion of the highly metastatic breast cancer cell line MDA-MB-231 partly through suppression of the Akt pathway. Int J Oncol 45: 1629-1637, 2014. 